\title{
Influence of Particle Size on Optical Constants From Pellets Measured With Terahertz Pulsed Spectroscopy
}

\author{
Tiphaine Bardon, Robert K. May, Philip F. Taday, and Matija Strlič
}

\begin{abstract}
Particle size is shown to influence both the overall value and the measurement precision of the refractive index and absorption coefficient of a sample diluted with high density polyethylene (HDPE) powder and compressed in a pellet. However, excessive grinding of a sample before it is mixed with HDPE powder can result in the formation of large particle clusters. These aggregates can induce internal scattering of the terahertz pulse within the pellet as well as lead to different mechanical properties of the pellet, which may change the distribution of air pores. These phenomena result in extracted optical constants with low accuracy and precision. The present work introduces a simple experimental procedure to improve the precision and accuracy of optical constants from spectroscopic pellets. This procedure consists of moderately grinding the sample powder in fine HDPE powder with a pestle and mortar. The standard deviation of the refractive index of Prussian blue has been observed to reduce from 0.14 to 0.02 following this procedure.
\end{abstract}

Index Terms-Particle size, pellet, Prussian blue, pulsed spectroscopy, scattering, sucrose, terahertz.

\section{INTRODUCTION}

$\mathbf{T}$ ERAHERTZ metrology is rapidly developing with the increased access to terahertz technologies. Terahertz timedomain spectroscopy (TDS) is widely used as it represents a powerful diagnostic tool to identify inter-molecular and some intra-molecular vibrations in different materials, as well as determine their optical constants. Refractive index and absorption coefficient are crucial parameters which enable better interpretation of terahertz images of layered structures such as coated pharmaceutical tablets [1], [2] or painted artefacts [3], [4]. For some materials absorbing in the terahertz region, extraction of optical constants from transmission through a solid slab or sheet may prove to be difficult due to excessive losses in the signal transmitted through a thick slab, and/or due to the etalon effect arising from multiple internal reflections of terahertz pulses on

Manuscript received September 28, 2015; revised January 21, 2016; accepted February 27, 2016. Date of publication April 20, 2016; date of current version June 14, 2016. This work was supported by the National Archief, The Hague, The Netherlands, and by the U.K. Engineering and Physical Research Council Doctoral Training Centre in Virtual Environments, Imaging and Visualisation under . (Corresponding author: Tiphaine Bardon.)

T. Bardon and M. Strlič are with the UCL Institute for Sustainable Heritage, The Bartlett, UCL Faculty of the Built Environment, London, WC1H 0NN, U.K. (e-mail: tiphaine.bardon.11@ucl.ac.uk).

R. K. May and P. F. Taday are with TeraView Ltd, St John's Innovation Park, Cambridge CB4 0DS, U.K. (e-mail: philip.taday@teraview.com).

Color versions of one or more of the figures in this paper are available online at http://ieeexplore.ieee.org.

Digital Object Identifier 10.1109/TTHZ.2016.2538641 thin sheet surfaces. To overcome these issues, a granular form of the material is often diluted into a low absorbing material, such as polyethylene powder, and the powder mixture then compressed into a thick pellet [5], [6]. Previous studies have investigated the influence of the particle size of granular materials on the spectral signature (absorbance, transmittance, absorption or attenuation coefficient spectra) of the material in the terahertz region, observing changes in the monotonic increase of the baseline, anomalous spectral features and distortions of vibrational absorption bands [7]-[12]. A study has recently suggested a curve-fitting equation to distinguish the absorption behavior of the material from scattering-induced effects in heterogeneous and scattering specimens [12]. Yet, no study has been made of the influence of particle size on refractive index values extracted using terahertz TDS. Additionally, few studies present data from duplicate or triplicate measurements (or more) and discuss the precision of the optical constants [13]-[19]. Many substances commonly studied with terahertz TDS are white, therefore the distribution of their particles within the white polyethylene matrix is difficult to observe. The study of optical properties of pigments in the terahertz region has enabled us to observe the influence of particle size as well as of the mixing procedure of the granular material on the homogeneity of spectroscopic pellets and on the overall value and precision of the optical constants. These observations are reported and discussed in the present letter, together with best practice of pellet preparation.

\section{Materials AND Methods}

\section{A. Spectroscopic Pellets}

Sucrose is a chemical readily available in large quantities and has a distinct spectral signature in the terahertz region $0.15-3 \mathrm{THz}$ [20]. Sucrose powder, therefore, is a convenient material for investigating the influence of particle size on the measured optical constants, as large amounts can be processed to obtain sufficient quantities of each size range of interest for spectroscopic analysis. Prussian blue is regarded as the first modern synthetic pigment and was widely used in artworks and documents due to its availability and ease of use [21]. The strong contrast in color between the white polyethylene matrix and Prussian blue facilitates the observation of the effects of sample preparation on the homogeneity of the pellet.

Powders of sucrose with different grain sizes were prepared as described elsewhere [7] in five categories: $53-75,75-106$, $106-150,150-250$, and $>250 \mu \mathrm{m}$. Powders from each size category were then mixed with a low-absorbing HDPE powder 
(Inducos 13/1, with particle size $<80 \mu \mathrm{m}$ ) with a mass ratio of sucrose to polyethylene of $10 \%$.

Prussian blue pigment (L. Cornelissen \& Son, London, U.K.) was dispersed on a glass slide and examined under optical microscope: individual particles could not be resolved with the optical microscope, as observed by Eastaugh et al. [22], but the average size of particle aggregates was found to be below $13 \mu \mathrm{m}$. Prussian blue was mixed with HDPE powder with a mass ratio of pigment to polyethylene of $10 \%$, using three different procedures. Procedure a consisted of further grinding the pigment powder in an agate mortar before mixing it with HDPE powder. Procedure $b$ consisted of mixing the pigment powder as received with HDPE (the same procedure as the one used for the preparation of sucrose pellets). Procedure $c$ consisted of moderately grinding the powder mixture obtained from Procedure $b$ using a pestle and mortar until a homogeneous blue powder was obtained. Procedures $b$ and $c$ therefore have the same particle size of pigment powder before mixing with HDPE powder, but the mixing procedure is different. Each sample powder mixture was pressed into three $400-\mathrm{mg}$ pellets using a hydraulic press (Specac, P/N 15011) under 2 tons of pressure for an equal amount of time, resulting in triplicate pellets with a diameter of $13 \mathrm{~mm}$ and a thickness of 3.53-3.87 $\mathrm{mm}$ (depending on sample and mixture preparation).

\section{B. Terahertz Time-Domain Spectroscopy}

Prepared pellets were spectroscopically analyzed in transmission mode using a commercially available time-domain spectrometer, TPS Spectra 3000 (TeraView Ltd., Cambridge, U.K.). The pellets were placed in a nitrogen purged sample compartment, at the focal plane of a focused terahertz beam. The diameter of the terahertz beam at the focal point was measured by placing a variable diameter circular aperture at the focal plane of the beam and by reducing the aperture diameter until the beam path begins to be blocked, i.e., an offset in the baseline of the absorbance spectrum is observed compared to the baseline when the beam path is unblocked. The results indicate a beam diameter of $6.5 \mathrm{~mm}$ at $1.2 \mathrm{THz}$ and $3.5 \mathrm{~mm}$ at $3 \mathrm{THz} .1800$ scans were acquired at a scan rate of $30 \mathrm{~Hz}$, and co-averaged to minimize random noise (i.e., each time point of the resulting waveform is the average of the 1800 points acquired at the same time position). The Fourier transform of the co-averaged waveforms resulted in a working spectral range of $5-100 \mathrm{~cm}^{-1}$ for sucrose, and $5-70 \mathrm{~cm}^{-1}$ for the highly absorbing Prussian blue, with a spectral resolution of $1.2 \mathrm{~cm}^{-1}$. The signal transmitted through a 360-mg pellet of pure polyethylene powder was used as a reference measurement. Different reference pellets were prepared and used for different days of acquisition, and their thickness ranged from 3315.7 to $3361 \mu \mathrm{m}$.

\section{Refractive Index Calculations}

Constant (i.e., frequency-independent) refractive indices were calculated as follows, and reported in Table I

$$
n_{\text {Constant }}=1+\frac{2 \Delta t}{d}
$$

TABLE I

Optical Properties and Thicknesses of Spectroscopic Pellets

\begin{tabular}{|c|c|c|c|c|}
\hline Sample & $\begin{array}{l}\text { "Constant" } \\
\text { refractive } \\
\text { index }\end{array}$ & $\begin{array}{l}\text { Slope of the } \\
\text { absorption } \\
\text { coefficient }\end{array}$ & $\begin{array}{c}\text { Time } \\
\text { delay } \Delta t \\
(\mu \mathrm{m})\end{array}$ & $\begin{array}{c}\text { Difference } \\
\text { in } \\
\text { thickness } \\
d(\mu \mathrm{m})\end{array}$ \\
\hline Sucrose $>250 \mu \mathrm{m}$ & $1.77 \pm 0.10$ & $6.76 \pm 1.14$ & $68 \pm 11$ & $176 \pm 15$ \\
\hline Sucrose $150-250 \mu \mathrm{m}$ & $1.84 \pm 0.08$ & $1.59 \pm 0.35$ & $78 \pm 7$ & $186 \pm 22$ \\
\hline Sucrose $106-150 \mu \mathrm{m}$ & $1.79 \pm 0.01$ & $0.72 \pm 0.28$ & $81 \pm 6$ & $205 \pm 16$ \\
\hline Sucrose $75-106 \mu \mathrm{m}$ & $1.71 \pm 0.01$ & $0.38 \pm 0.04$ & $74 \pm 3$ & $209 \pm 6$ \\
\hline Sucrose $53-75 \mu \mathrm{m}$ & $1.74 \pm 0.06$ & $0.28 \pm 0.12$ & $78 \pm 3$ & $212 \pm 9$ \\
\hline Prussian blue a & $1.87 \pm 0.14$ & $1.39 \pm 0.51$ & $148 \pm 21$ & $342 \pm 15$ \\
\hline Prussian blue b & $1.75 \pm 0.05$ & $0.78 \pm 0.12$ & $137 \pm 8$ & $363 \pm 5$ \\
\hline Prussian blue $\mathrm{c}$ & $1.48 \pm 0.02$ & $0.23 \pm 0.03$ & $131 \pm 6$ & $550 \pm 5$ \\
\hline
\end{tabular}

Average "constant" refractive index, slope of the absorption coefficient, observed time delay and measured difference in thickness between the sample and reference pellets, and their associated standard deviations for each set of triplicate pellets. The "constant" refractive index and the slope of the absorption coefficient were calculated using (1) and the linear fit to (4) in the range $5-25 \mathrm{~cm}^{-1}(R=0.98 \pm 0.01)$.

where $\Delta t$ is the time difference (in units of $\mathrm{mm}$ ) between the maxima of the waveforms collected for the sample and the reference pellets, and $d$ is the difference in thickness (in units of $\mathrm{mm}$ ) between the sample and reference pellets. While this value is a good and easy to compare indicator of the delay of the pulse due to changes in speed of propagation rather than to changes in pellet thickness, this value would only truly be a constant if the speed of propagation were also constant, i.e., did not depend on frequency, with no broadening of the pulse observed after transmission through the pellet. This is often not the case.

Therefore, frequency-dependent refractive indices were also calculated and are presented in Figs. 1 and 4(b). These indices were calculated using the unwrapped phase $\phi(\nu)$ of the complex transmittance $T(\nu)$, which is the ratio of the complex-valued sample and reference spectra, $S(\nu)$ and $R(\nu)$, respectively [23]

$$
\begin{aligned}
& T(\nu)=\frac{S(\nu)}{R(\nu)}=T_{0} e^{i \phi(\nu)}=T_{0} e^{i\left(\phi_{s}(\nu)-\phi_{R}(\nu)\right)} \\
& n(\nu)=1-\frac{\phi(\nu)}{2 \pi \nu_{c m^{-1}} d_{c m}} .
\end{aligned}
$$

If the sample and reference time-domain waveforms are not aligned on the time axis, a phase offset $\Delta \phi(\nu)$ proportional to the initial time difference is subtracted to $\phi(\nu)$ for the calculation of the refractive index.

Attenuation of the amplitude of the terahertz electric field transmitted through the sample pellet compared to the electric field transmitted through the reference pellet arises from three phenomena: 1) absorption by the sample; 2) Fresnel reflection losses at the surface of the pellet; and 3) internal scattering from powder particles and air pores. Frequency-dependent absorption coefficients presented in Figs. 2 and 4(a) were calculated from (3) using the following formula correcting for the effects of Fresnel reflection losses [23]:

$$
\alpha(\nu)=\frac{2}{d_{\mathrm{cm}}}\left(\ln \left[\left|\frac{R(\nu)}{S(\nu)}\right|\right]+\ln \left[\frac{4 n_{\mathrm{Ref}} n(\nu)}{\left(n(\nu)+n_{\mathrm{Ref}}\right)^{2}}\right]\right)
$$

with $n_{\text {Ref }}$ set to 1 . Equation (4) does not correct for any scattering losses originating from within the pellet. Therefore, $\alpha(\nu)$ 


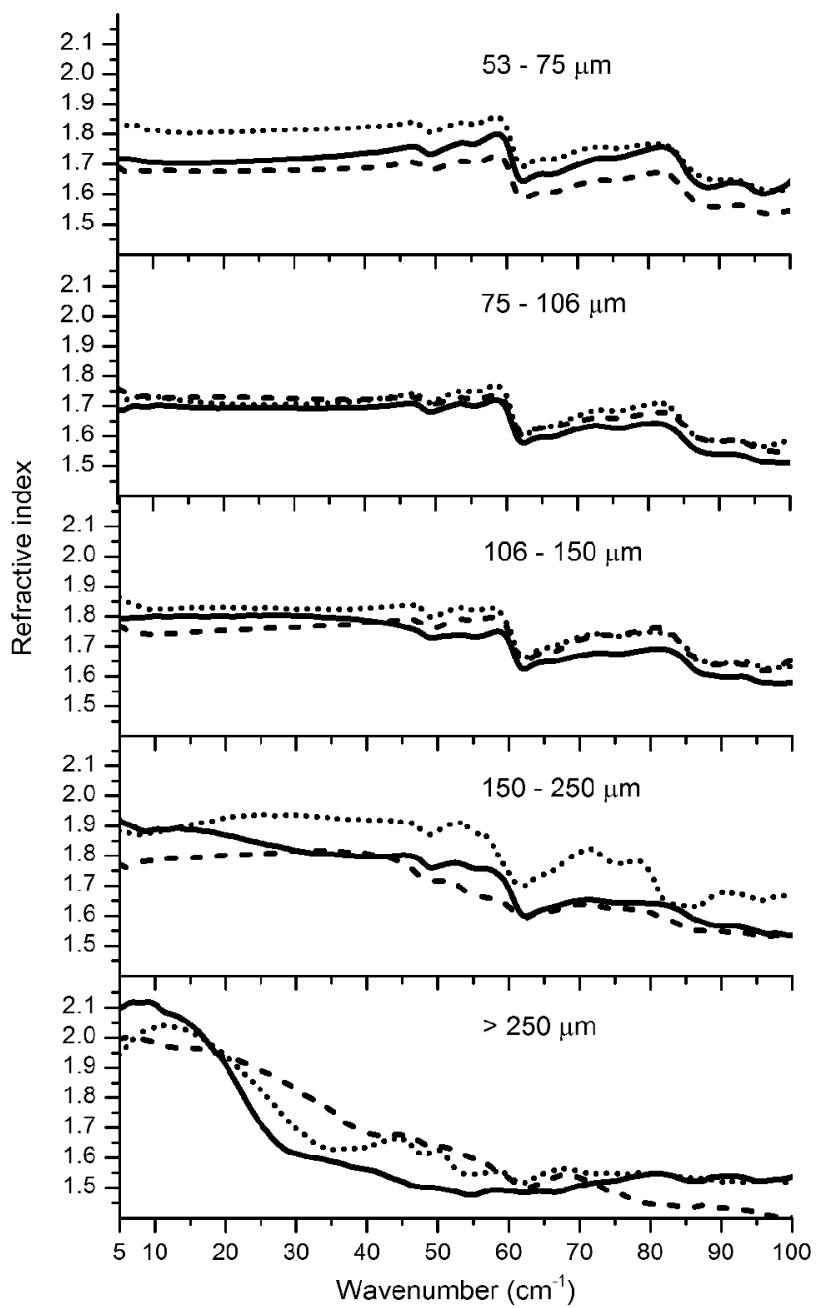

Fig. 1. Frequency-dependent refractive indices of pellets of sucrose powders with different grain sizes, calculated using (3). Solid, dashed, and dotted lines in each graph represent data collected from each triplicate pellet.

is indicative of the total extinction of the terahertz beam, and encompasses any absorption by the sample powder, as well as scattering from powder particles and air pores.

\section{Thickness Measurements}

Using (1), it can be observed that the error in refractive index $\Delta n$ caused by an error $\Delta d$ in the reading of the thickness of the pellets is a function of $d$ :

$$
\Delta n=|n(d+\Delta d)-n(d)|=\frac{\Delta d}{d+\Delta d} \frac{2 \Delta t}{d} .
$$

In this study, $d$ was found to vary between $164-553 \mu \mathrm{m}$. For a sucrose pellet with a difference in thickness between the sample and reference pellets of $d=164 \mu \mathrm{m}$, a time difference $\Delta t=$ $54.8 \mu \mathrm{m}$ was observed. Uncertainties in readings of $d$ of 1 and $10 \mu \mathrm{m}$ would correspond to uncertainties in refractive index of

$$
\begin{aligned}
& \frac{\Delta n_{0.01}}{n_{\text {Constant }}}=\frac{0.0384}{1.6683}=2.3 \%, \quad \text { for } \Delta d=10 \mu \mathrm{m} \\
& \frac{\Delta n_{0.001}}{n_{\text {Constant }}}=\frac{0.0041}{1.6683}=0.2 \%, \quad \text { for } \Delta d=1 \mu \mathrm{m} .
\end{aligned}
$$

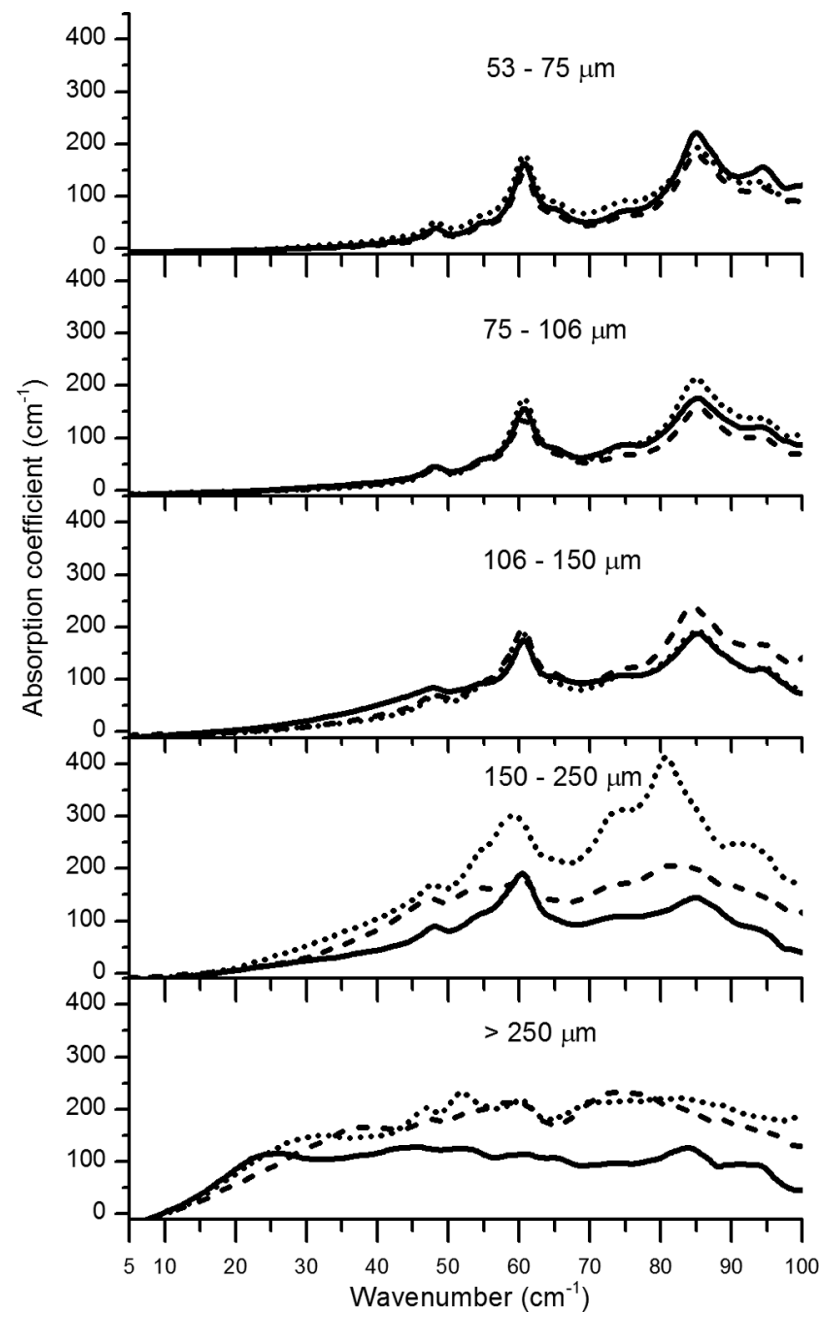

Fig. 2. Frequency-dependent absorption coefficients of pellets of sucrose powders with different grain sizes, calculated using (4). Solid, dashed, and dotted lines in each graph represent data collected from each triplicate pellet.

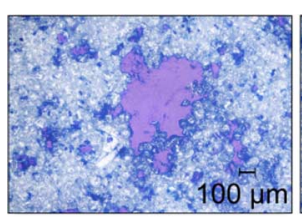

(a)

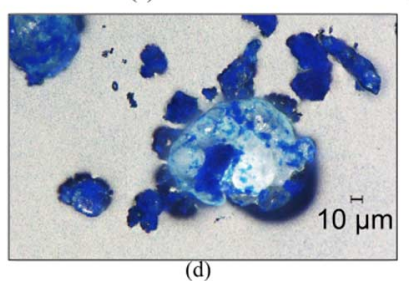

(b)

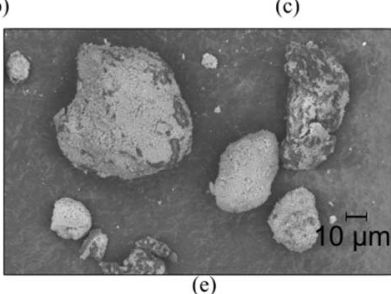

Fig. 3. (a)-(c) Microscope images of the surface of spectroscopic pellets prepared following Procedures $a, b$, and $c$, respectively (magnification: $\times 200$ ); (d) microscope image (magnification: $\times 1000$ ); and (e) SEM image of the powder mixture of Prussian blue and HDPE produced following Procedure $c$. On the SEM image produced with back scattered electrons, Prussian blue appears as bright, as it contains iron ions and is therefore heavier than HDPE.

For the same thickness difference of $d=164 \mu \mathrm{m}$, a material with a larger refractive index than sucrose would lead to a longer optical path length $n * d$, and therefore to a larger recorded 

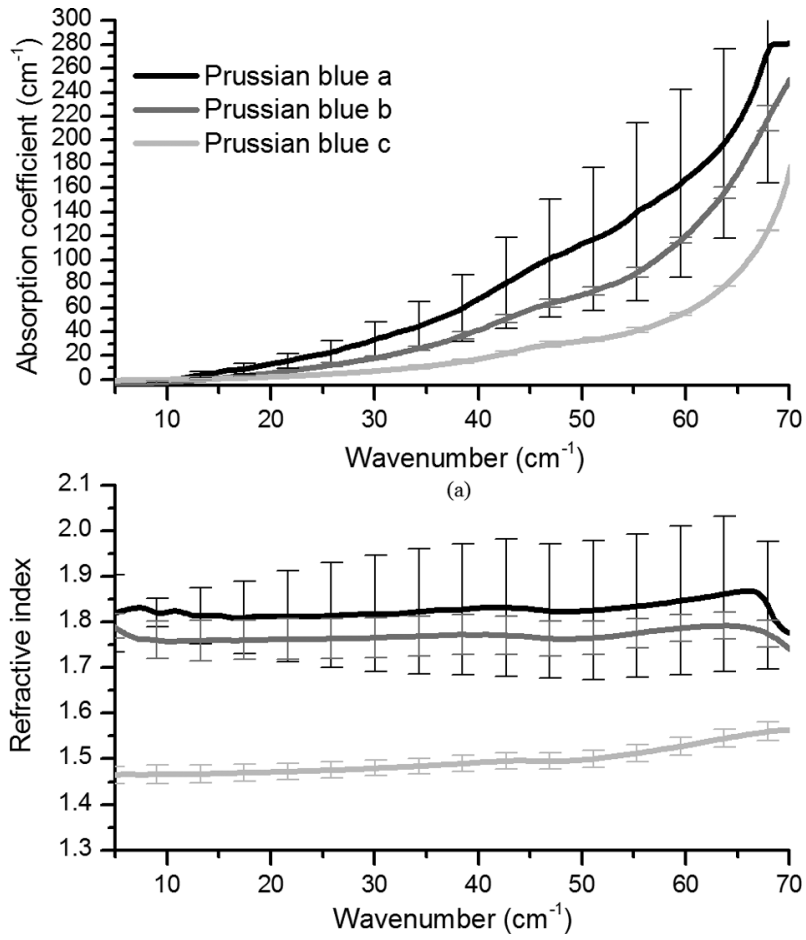

(b)

Fig. 4. Frequency-dependent absorption coefficients (a) and refractive indices (b) of Prussian blue spectroscopic pellets, prepared following Procedures $a, b$, and $c$ (black, gray and light gray lines, respectively). The lines represent the average of data from triplicate pellets, and the error bars represent the standard deviation

time delay. For such material, the variation $\Delta n$ due to a thickness accuracy $\Delta d$ would therefore be even larger than the values reported here. To minimize uncertainties in $n$ due to thickness measurements, a caliper with an accuracy of $\pm 1 \mu \mathrm{m}$ was selected, and calculations of $n$ were made using the average of triplicate thickness measurements on each pellet.

\section{E. Microscopy}

To observe particle sizes and pigment distribution, powder mixtures of Prussian blue prepared following Procedure $c$ were dispersed onto a conductive carbon adhesive tape and imaged with a Hitachi TM 3030 scanning electron microscope (SEM). Additionally, the surface of the tape as well as the surface of spectroscopic pellets of Prussian blue were imaged with the $3 \mathrm{D}$ VHX-5000 microscope (Keyence).

\section{RESULTS AND DISCUSSION}

Walther et al. [20] reported that the refractive index of a solid spectroscopic pellet obtained from milled sucrose powder diluted with polyethylene is 1.8 on average over the frequency range $0.2-4 \mathrm{THz}$. While Fig. 1 and Table I report similar values, they also indicate that the average value and precision of the refractive index measurements from such pellet depends on the particle size range. While the average value and standard deviation of the refractive index is lower for particles with sizes between 75 and $106 \mu \mathrm{m}$, both are seen to increase with larger particle sizes, until reaching a standard deviation of 0.1 for particles larger than $250 \mu \mathrm{m}$. A similar phenomenon is observed for the absorption coefficients in Fig. 2: for particles larger than
$75 \mu \mathrm{m}$, the increasing baseline is more pronounced and less reproducible with increasing particle size. This is particularly evident when approximating the power-law frequency dependence of the baseline of the absorption coefficient to a linear dependence at low frequencies: the slope of the linear fit of the absorption coefficient between $5-25 \mathrm{~cm}^{-1}$ increases with increasing particle size, together with its standard deviation, as reported in Table I.

Additionally, for both refractive index and absorption coefficient values, spectral features at 48, 55, 60, 74, 85 and $94 \mathrm{~cm}^{-1}$ characteristic of sucrose at $300 \mathrm{~K}$ [20] are well observed in all triplicate spectra for particles with sizes below $150 \mu \mathrm{m}$. However, for larger particles these spectral features broaden and are not consistently observed. For particles larger than $250 \mu \mathrm{m}$, additional features are also detected. When re-analyzing pellets with a sucrose particle size larger than $150 \mu \mathrm{m}$, rotating them in the plane perpendicular to the direction of propagation of the beam, these additional bands were observed not to be repeatable. Such spectral artifacts and distortions, together with increasing baselines, were also observed by Shen et al. [7] for sucrose particles with sizes more than $250 \mu \mathrm{m}$ in a powder cell, and by Franz et al. [8] for phenyllactic acid particles with sizes between 400-600 $\mu \mathrm{m}$, diluted with PE and compressed into pellets. These studies describe spectral artifacts and distortions as a result of scattering, induced by the refractive index discrepancy between a sample particle with a size similar to the incident wavelength (100-2000 $\mu \mathrm{m}$ in dry air) and the surrounding polyethylene matrix, also known as the Christiansen effect. For particles smaller than the incident wavelength, the media constituting the pellet can be considered homogeneous, and the terahertz beam is not scattered. Such scattering can also influence the time delay $\Delta t$ between the terahertz pulses transmitted through the reference and sample pellets: a terahertz pulse experiencing an internal reflection on a large particle will escape the spectroscopic pellet at a later time than a pulse experiencing no internal reflection, and the measured $\Delta t$ would be larger. Yet, if multiple internal scattering occurs on various large particles, the incident signal would be separated into different scattered signals escaping the same pellet at different times, and with different intensities. These signals would overlap and the resulting waveform would have a Full Width at Half Maximum (FWHM) and a time position of the maximum amplitude (i.e., a time delay) depending on the number and degree of overlap of these scattered signals. This is the reason why the average time delays for sucrose pellets reported in Table I do not display a systematic decrease with smaller particle size, while their standard deviation do. The change in the degree of overlap of the scattered signals would change the shape of the waveform, and therefore its spectral dependency in the frequency-domain: this is why additional spectral features may be observed for pellets containing particles with sizes similar to or larger than the wavelength of the incident pulse. When re-analyzing pellets with a sucrose particle size larger than $150 \mu \mathrm{m}$, rotating them about the axis of propagation of the pulse, the orientation of the sucrose particle-HDPE particle interfaces is changed with respect to the polarized incident beam. Therefore, the incident pulse may not undergo the same multiple internal scattering and the number and degree of overlap of the scattered signals may change: the 
shape of the resulting waveform may differ and as a result, the spectral features observed once for this same pellet are not observed again. Therefore, while we can consider that pellets constituted of large particles of sucrose are birefringent, that does not mean that sucrose itself is birefringent.

It should be noted that the standard deviation for both refractive index and absorption coefficient is larger for particle sizes comprised between 53-75 $\mu \mathrm{m}$ than for particle sizes between 75-106 $\mu \mathrm{m}$. This could be explained by the fact that at a critical particle size, the active surface area of a particle is such that surface forces (e.g., electrostatic or van der Waals) start to be significant and sample particles may have a preferred affinity to bind with other sample particles, rather than with HDPE particles. This would create clusters of sample particles which can be assimilated to large sample particles and would lead to scattering effects similar to those mentioned above.

Prussian blue fine particles are known to form aggregates [22] and unlike white sucrose particles are intensely colored, which makes it possible to observe clusters of particles in the white HDPE matrix. Fig. 3(a) shows an example of such a cluster at the surface of a spectroscopic pellet: the fine Prussian blue powder was further ground prior to its mixing with HDPE and this resulted in the formation of aggregates which can be larger than $500 \mu \mathrm{m}$. Mixing the Prussian blue powder without additional grinding resulted in aggregates smaller than $150 \mu \mathrm{m}$, as observed in Fig. 3(b). The use of mechanical forces through moderate grinding of the powder mixture with a pestle and mortar to counterbalance the electrostatic forces at the surface of small Prussian blue particles resulted in an intricate binding of Prussian blue particles with HDPE particles, as observed in Fig. 3(d) and (e). This procedure produced a homogeneous powder, and the resulting spectroscopic pellet could be considered as a homogeneous medium with respect to the wavelength of the incident beam, as observed in Fig. 3(c).

Refractive indices and absorption coefficients from Prussian blue pellets produced using the different procedures are listed in Table I and Fig. 4. Standard deviations reported in this figure clearly indicate that optical constant values extracted from pellets produced with Procedure $c$ are significantly more reproducible than values extracted from pellets produced with Procedure $a$. Furthermore, as observed for sucrose with increasing particle sizes, the increasing baseline due to scattering with the Christiansen effect is also more pronounced in absorption coefficients extracted from pellets resulting from Procedure $a$ and $b$. Finally, Fig. 4(b) and Table I indicate that the average refractive indices for pellets resulting from Procedure $a$ and $b$ have an offset of 0.3 to 0.4 compared to refractive indices from homogeneous pellets. Table I also indicates that pellets resulting from Procedure $c$ were found to be significantly thicker than pellets with a poor pigment distribution. One would expect that this would increase their associated time delays, yet Table I reports decreased time delays. Analyzing the time-domain waveforms (data not shown here), the signals from Procedures $a$ and $b$ are more attenuated and have a broader full-width at half-maximum (FWHM) with respect to their reference signals than the signals from Procedure $c$. These three signs imply that the optical path for inhomogeneous pellets prepared with Procedures $a$ and $b$ is increased by internal scattering, compared to homogeneous pellets prepared with Procedure $c$. Multiple internal scattering within the pellet would induce multiple time delays collected for the same incident pulse, which increases the FWHM of the resulting signal. Prussian blue from other suppliers have also been analyzed (data not shown here). Using Procedure c, homogeneous pellets of similar thickness difference and time delays than those shown for "Prussian blue c" in this study were obtained. As a result, refractive index and absorption coefficient values were found to be comparable. This strengthens the argument that internal scattering on clusters of particles with a size similar to or superior to the wavelength of the incident pulse together with difference in pellet thickness due to different responses to compression or expansion depending on particle distribution are at the origin of the large difference in optical properties between the pellets prepared following different procedures.

This work introduces a simple experimental procedure to minimize scattering from particles so as to improve the precision (i.e., reduce random errors) of the optical constants extracted from diluted spectroscopic pellets. Its efficiency is particularly noticeable for powders with strong surface forces and prone to aggregate, such as Prussian blue as reported here. Yet scattering may also occur due to the presence of air pores of sizes similar to the incident wavelength and embedded in a homogeneous matrix. Different powders would present different mechanical properties once diluted with HDPE and pressed into a pellet, and the number and size of the pores within a pellet may differ depending on the mixing procedures (as observed here for Prussian blue) and on the nature of the sample material (its compressive strength and expansion properties, its hygroscopicity, the nature of its surface forces). More generally, optical constants extracted from porous pellets may contain an offset compared to those extracted from solid materials, the size of which would depend on the size and number of pores within the pellet. The use of Kramers-Kronig equations [24] and effective medium theory [10], [13] to account for scattering from air pores within the pellet could further help to obtain accurate and comparable optical constants from spectroscopic pellets. Finally, it should be noted that this procedure may not be used for materials prone to phase transformation during grinding or blending with a pestle and mortar.

\section{REFERENCES}

[1] L. Maurer and H. Leuenberger, "Terahertz pulsed imaging and near infrared imaging to monitor the coating process of pharmaceutical tablets," Int. J. Pharm., vol. 370, no. 1-2, pp. 8-16, Mar. 2009.

[2] L. Ho, R. Müller, K. C. Gordon, P. Kleinebudde, M. Pepper, T. Rades, Y. Shen, P. F. Taday, and J. A. Zeitler, "Terahertz pulsed imaging as an analytical tool for sustained-release tablet film coating," Eur. J. Pharm. Biopharm., vol. 71, no. 1, pp. 117-23, Jan. 2009.

[3] A. J. L. Adam, P. C. M. Planken, S. Meloni, and J. Dik, "Terahertz imaging of hidden paint layers on canvas," Opt. Express, vol. 17, no. 5, pp. 3407-16, Mar. 2009.

[4] J. B. Jackson, J. Labaune, G. Mourou, I. N. Duling, G. Walker, J. Bowen, and M. Menu, "Terahertz pulse imaging of stratified architectural materials for cultural heritage studies," in O3A: Optics for Arts, Architecture, and Archaeology III, 2011, vol. 8084, Art. no. 808409.

[5] K.-E. Peiponen, J. A. Zeitler, and M. Kuwata-Gonokami, Terahertz Spectroscopy and Imaging, K.-E. Peiponen and M. Kuwata-Gonokami, Eds. Berlin, Germany: Springer-Verlag, 2013, vol. 171.

[6] P. U. Jepsen, D. G. Cooke, and M. Koch, "Terahertz spectroscopy and imaging-modern techniques and applications," Laser Photon. Rev., vol. 5 , no. 1, pp. 124-166, Jan. 2011 
[7] Y. C. Shen, P. F. Taday, and M. Pepper, "Elimination of scattering effects in spectral measurement of granulated materials using terahertz pulsed spectroscopy," Appl. Phys. Lett., vol. 92, no. 5, 2008, Art. no. 051103 .

[8] M. Franz, B. M. Fischer, and M. Walther, "The Christiansen effect in terahertz time-domain spectra of coarse-grained powders," Appl. Phys. Lett., vol. 92, no. 2, 2008, Art. no. 021107.

[9] J. R. Fletcher, G. P. Swift, D. C. Dai, J. a. Levitt, and J. M. Chamberlain, "Propagation of terahertz radiation through random structures: An alternative theoretical approach and experimental validation," J. Appl. Phys., vol. 101, no. 1, 2007, Art. no. 013102.

[10] L. M. Zurk, B. Orlowski, D. P. Winebrenner, E. I. Thorsos, M. LeahyHoppa, and M. R. Hayden, "Terahertz scattering from granular material," J. Opt. Soc. Amer. B, vol. 24, no. 9, pp. 2238-2243, 2007.

[11] K. Yamamo, K. Fukunaga, M. Mizuno, I. Hosako, H. Sotobayashi, and S. Fiorentino, "Examinat tion of pigments by using FT-THz," in 35th Int. Conf. on Infrared Millim. THz Waves (IRMMW-THz), 2010, pp. 6-7.

[12] V. V. Kornienko, R. a. Akhmedzhanov, I. E. Ilyakov, A. D. Mishin, P. a. Prudkovskii, O. V. Samotokhin, B. V. Shishkin, and G. K. Kitaeva, "Scattering effects in terahertz wave spectroscopy of granulated solids," IEEE Trans. THz Sci. Technol., vol. 5, no. 4, pp. 1-8, Jul. 2015.

[13] E. P. J. Parrott, J. A. Zeitler, and L. F. Gladden, "Accurate determination of optical coefficients from chemical samples using terahertz time-domain spectroscopy and effective medium theory," Opt. Lett., vol. 34, no. 23, pp. 3722-3724, 2009.

[14] P. U. Jepsen, U. Møller, and H. Merbold, "Investigation of aqueous alcohol and sugar solutions with reflection terahertz time-domain spectroscopy," Opt. Express, vol. 15, no. 22, pp. 14717-37, Oct. 2007.

[15] B. Fischer, M. Hoffmann, H. Helm, G. Modjesch, and P. U. Jepsen, "Chemical recognition in terahertz time-domain spectroscopy and imaging," Semicond. Sci. Technol., vol. 20, no. 7, pp. S246-S253, Jul. 2005.

[16] E. Berry, A. J. Fitzgerald, N. N. Zinov, G. C. Walker, S. Homer-Vanniasinkam, C. D. Sudworth, R. E. Miles, J. M. Chamberlain, and M. A. Smith, "Optical properties of tissue measured using terahertz pulsed imaging," in Proc. SPIE: Med. Imaging 2003: Phys. Med. Imaging, 2003, pp. 459-470.

[17] L. Duvillaret, F. Garet, and J.-L. Coutaz, "A reliable method for extraction of material parameters in terahertz time-domain spectroscopy," IEEE J. Sel. Top. Quantum Electron., vol. 2, no. 3, pp. 739-746, May/ Jun. 1996.

[18] W. Withayachumnankul, B. M. Fischer, H. Lin, and D. Abbott, "Uncertainty in terahertz time-domain spectroscopy measurement," J. Opt. Soc. Amer. B, vol. 25, no. 6, pp. 1059-1072, May 2008.

[19] M. Bernier, F. F. Garet, and J.-L. L. Coutaz, "Precise determination of the refractive index of samples showing low transmission bands by THz time-domain spectroscopy," IEEE Trans. THz Sci. Technol., vol. 3, no. 3, pp. 295-301, May 2013.

[20] M. Walther, B. M. Fischer, and P. U. Jepsen, "Noncovalent intermolecular forces in polycrystalline and amorphous saccharides in the far infrared," Chem. Phys., vol. 288, pp. 261-268, 2003.

[21] J. Kirby and D. Saunders, National Gallery Company Ltd., London, U.K., "Fading and colour change of Prussian blue: Methods of manufacture and the influence of extenders," National Gallery Tech. Bull., vol. 25, pp. 73-99, 2004 [Online]. Available: http://www.nationalgallery.org.uk/technical-bulletin/kirby saunders 2004

[22] R. Siddall, T. Chaplin, V. Walsh, and N. Eastaugh, Pigment Compendium. Routledge, U.K.: Taylor \& Francis, 2008.

[23] W. Withayachumnankul and M. Naftaly, "Fundamentals of measurement in terahertz time-domain spectroscopy," J. Infrared Millim. THz Waves, vol. 35, pp. 610-637, 2014.

[24] H. Tuononen, E. Gornov, J. a. Zeitler, J. Aaltonen, and K.-E. Peiponen, "Using modified Kramers-Kronig relations to test transmission spectra of porous media in THz-TDS," Opt. Lett., vol. 35, no. 5, pp. 631-3, Mar. 2010.

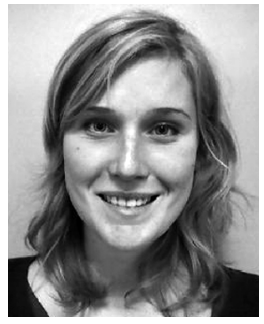

Tiphaine Bardon received the M.Sc. degree in physics and nanosciences from PHELMA, Grenoble-INP Engineering School, France, in 2011, the MRes Heritage Science at The UCL Institute for Sustainable Heritage, London, UK, in 2012, and is currently working toward the Eng.D. degree at the Institute. Her doctoral research focuses on terahertz time-domain spectroscopy and imaging as a diagnostic and imaging tool for the study of archival documents.

Her research interests include heritage science, spectroscopic imaging and non-invasive techniques.

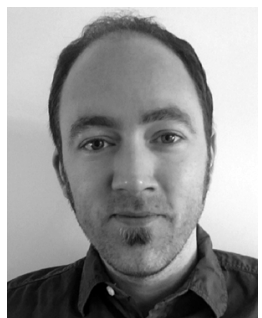

Robert K. May received B.Sc. (Hons) and Ph.D. degrees in experimental physics from the National University of Ireland Maynooth in 2002 and 2008, respectively.

From 2008 to 2010 he was a Postdoctoral Research Associate with the Terahertz Applications Group, Department of Chemical Engineering at the University of Cambridge, U.K. Since 2010, he has been an Application Scientist at TeraView Ltd., Cambridge, U.K. His research interests lie in the development of non-destructive, terahertz sensing techniques for industrial applications, in particular for coating applications.

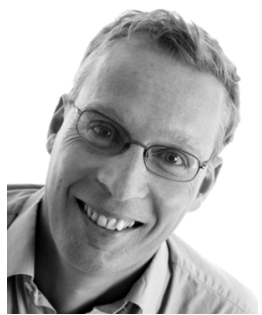

Philip F. Taday received the B.S. degree in physics and the Ph.D. degree in chemistry from the University of Reading, U.K., in 1991 and 1996, respectively.

When working at the Central Laser Facility at the CLRC Rutherford Appleton Laboratory, he developed a femtosecond tabletop laser system as well as worked on novel tunable sources. In August $2000 \mathrm{Dr}$ Taday began working at Toshiba Research Europe Limited, of Cambridge, England, on terahertz spectroscopy. In April of 2001 this terahertz program was spun-out of Toshiba, becoming TeraView Ltd. He is currently the Principal Scientist and Head of the Applications at TeraView Ltd., Cambridge, U.K.

Dr Taday is a member of the Institute of Physics.

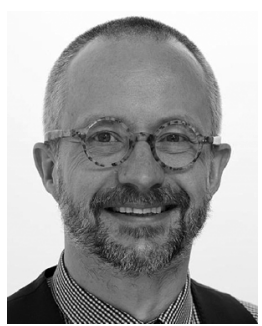

Matija Strlič received the Ph.D. degree in chemistry from the University of Ljubljana, in 2000.

He is Professor of Heritage Science and Course Director at The UCL Institute for Sustainable Heritage, London, U.K. His research focus is in the cross-disciplinary field of heritage science, particularly development of new scientific tools and methods to study heritage materials and collections, and their interactions with the environment. 